\title{
Pollutions chimique et bactériologique des eaux souterraines des exploitations maraîchères irriguées de la commune de Grand-Popo : cas des nitrates et bactéries fécales
}

\author{
C. Séraphin ATIDEGLA ${ }^{1^{*}}$ et K. Euloge AGBOSSOU ${ }^{2}$ \\ ${ }^{1}$ Centre Régional pour la Promotion Agricole (CeRPA) Atlantique - Littoral, BP 433 Abomey - Calavi, Bénin. \\ ${ }^{2}$ Faculté des Sciences Agronomiques (FSA), 01 BP 526 Cotonou, Bénin. \\ *Auteur correspondant, E-mail : eulser2000@yahoo.fr, agbossou2001@yahoo.fr
}

\section{RESUME}

La dégradation de la qualité des eaux constitue un phénomène très préoccupant dans le Sud-Bénin où se trouve la majorité des zones humides du pays. C'est le cas des Arrondissements d'Agoué et de Grand-Popo, où le maraîchage sous irrigation avec les eaux souterraines connait un essor depuis les années 90, coïncidant avec le début de la chute drastique de la production halieutique dans les eaux fluviales et marines (Atidégla, 2005). Les méthodes de collecte des données de terrain se résument aux observations directes et entretiens sur la base des questionnaires, aux prélèvements d'eaux sur les sites d'étude et aux analyses des échantillons dans le laboratoire de la Direction Générale de l'Eau. Les résultats des enquêtes révèlent que certaines activités humaines, liées ou non au maraîchage sous irrigation, sont responsables de la pollution des eaux. Ce sont: fertilisation des sols par les engrais chimiques et organiques; défécation sur les sites par les populations et les animaux en divagation; déversement de toutes sortes de déchets. Les teneurs moyennes en nitrates (135 mg/l) obtenues dépassent la norme de $45 \mathrm{mg} / \mathrm{l}$ préconisée par l'Organisation Mondiale de la Santé. Pour ce qui est des bactéries fécales, les nombres moyens en coliformes fécaux (78) et streptocoques fécaux (123) à GrandPopo dépassent la norme Béninoise de 0 germe par millilitre d'eau. A Agoué, nous avons eu respectivement (591) et (341). Ces résultats justifient la pollution desdites eaux par les nitrates et les bactéries fécales.

(c) 2010 International Formulae Group. All rights reserved.

Mots clés : Fertilisation, défécation, déchets, teneur, norme, pollution chimique, pollution bactériologique.

\section{INTRODUCTION}

Dans la plupart des pays côtiers de l'Afrique, les principales sources de revenu des populations reposent sur l'exploitation des écosystèmes côtiers en général et la pêche en particulier (Dossa et al., 2005). A Grand-Popo, ville côtière au Sud-Bénin, la production halieutique a fortement baissé depuis une décennie dans les eaux continentales et maritimes. L'alternative à cette situation est la pratique du maraîchage sous irrigation par une frange importante de la population (plus de $70 \%$ ), en dépit des problèmes liés au faible niveau de fertilité des terres du littoral et à l'insuffisance des superficies exploitables, due surtout à l'accélération de l'urbanisation (Atidegla, 2005). Les zones phares où s'exerce l'activité dans la Commune de Grand-Popo concernent deux Arrondissements: Grand-Popo et Agoué.

Le facteur favorisant l'exercice du maraîchage est l'accessibilité aisée à l'eau souterraine (la nappe phréatique étant située 
entre 3 et $6 \mathrm{~m}$ de la surface du sol) et l'exhaure de l'eau est faite à partir des motopompes ou des pompes électriques installées sur des puits traditionnels ou des forages. Les modes d'irrigation utilisés sont le type traditionnel avec arrosoir $(5 \%)$ et le mode amélioré (95\%), basé sur l'utilisation de l'énergie d'une pompe électrique ou d'une motopompe à essence, avec des tuyaux qui distribuent l'eau sous pression directement aux plantes par le biais des pommes d'arrosage. D'après Atinkpahoun, (2004), le Bénin recèle d'importantes ressources hydriques en ce qui concerne les eaux de surface et souterraines mais, le triste constat fait aujourd'hui est que, pratiquement toutes les régions du pays sont touchées par le problème de dégradation de la qualité et de la pollution des eaux. En effet, s'il est vrai que l'irrigation permet d'accroître les rendements et la diversification, il n'en demeure pas moins que des activités connexes et les mauvaises pratiques des populations aient des répercussions sur la qualité et la conservation des écosystèmes côtiers et de leurs ressources. C'est le cas des Arrondissements de Grand-Popo et d'Agoué, eu égard à l'essor que connaît le maraîchage sous irrigation marqué par une fumure excessive des sols à partir des apports abondants d'engrais (minéraux et organiques). En effet, la plupart de la population n'ayant pas accès au réseau de distribution d'eau potable de la SONEB (Société Nationale des Eaux du Bénin) utilise les eaux des puits ou forages pour la consommation et diverses activités domestiques. La pollution de ces eaux peut entraîner des maladies d'origine hydrique telles que la diarrhée, le choléra et le paludisme (Azonhè, 2008). Par conséquent, il est urgent d'évaluer la qualité des eaux de la zone qui ne sont pas à l'abri des pollutions chimique et bactériologique.

L'objectif de la présente étude est d'identifier les différentes sources de pollution, de déterminer le niveau de pollution des eaux souterraines de GrandPopo par les nitrates et les bactéries fécales, et d'évaluer l'impact potentiel de cette pollution sur la santé des populations et l'environnement.

\section{MATERIEL ET METHODES Milieu d'étude}

D'une superficie de $240 \mathrm{Km}^{2}$, la Commune de Grand-Popo (Figure 1) est située dans le Département du Mono, en bordure de la côte Atlantique. Les coordonnées géographiques de Grand-Popo sont: latitude $6^{\circ} 17^{\prime}$ Nord et longitude $1^{\circ} 49^{\prime}$ Est. Son altitude est de $5 \mathrm{~m}$ au-dessus de la mer. Elle s'étend sur une longueur de $35 \mathrm{~km}$ et abrite une population de 40335 habitants (INSAE, 2004). Comme toute la région côtière, elle jouit d'un climat de type subéquatorial, caractérisé par de faibles variations de température et par deux saisons sèches alternant avec deux saisons pluvieuses qui se répartissent comme suit : i) une grande saison sèche : novembre à avril, ii) une grande saison pluvieuse : avril à juillet, iii) une petite saison sèche : août à septembre, iv) une petite saison pluvieuse : septembre à octobre. La moyenne pluviométrique annuelle est de $922 \mathrm{~mm}$ et la température moyenne annuelle est de $27,7^{\circ} \mathrm{C}$. L'écart thermique moyen est de $5,8^{\circ} \mathrm{C}$, ce qui est relativement faible et tolérable à la vie des êtres vivants (Atidegla, 2005).

La Commune est située dans la zone agroécologique des pêcheries du Bénin comprenant le domaine littoral très complexe couvert en sa grande partie de sols sableux peu fertiles, mais aptes aux plantations de cocotiers (MAEP, 2005). De par leurs caractéristiques physico-chimiques, ils sont classés en deux grands groupes: les sols sableux et les sols alluviaux. Le paysage est caractérisé entre autres par des plantations de cocotiers (Cocos nucifera) en bordure de la mer et sur les surfaces cultivables, des savanes à Borassus aethiopum présentes sur les sols très sableux et bien drainants de même que quelques savanes à Mitragyna inermis qui caractérisent les aires inondables aux sols argileux. Les champs de culture et les zones de maraîchage disponibles représentent environ $40 \%$ du paysage. 
Le complexe hydrographique comprend notamment le fleuve Mono et ses affluents, la lagune de Grand-Popo, le fleuve Couffo et le lac Ahémé communiquant avec l'Océan par le Chenal Aho (Figure 2).

\section{Choix des villages et des enquêtés}

L'enquête exploratoire a permis de retenir dans la zone d'étude, six (6) villages des Arrondissements de Grand-Popo et d'Agoué, disposant d'exploitations maraîchères fonctionnelles toute l'année, et comme site témoin un village où l'activité n'est pas exercée. Les critères de choix des villages sont : i) Disposer d'au moins dix (10) exploitations maraîchères de plus de deux ans au minimum ; ii) Avoir les sites de part et d'autre de la Route Nationale Inter Etats 1 (RNIE 1) Cotonou-Lomè ; iii) Disposer par village, d'au moins dix maraîchers exerçant chacun sur une superficie supérieure ou égale à 0,5 ha.

Ainsi, les villages d'étude retenus sont: Ewé-Condji, Yodo-Condji, Nikoué-Condji dans l'Arrondissement de Grand-Popo, Zogbédji, Ayi-Guinnou et Agoué 1 dans l'Arrondissement d'Agoué, tandis que Hounsoukouè a été retenu au titre du village témoin (où ne se pratique pas le maraîchage sous irrigation) dans l'Arrondissement de Grand-Popo. Sur la base des résultats des enquêtes préliminaires, le nombre total d'exploitants des six (06) villages cibles peut être estimé à environ 200 parmi lesquels un échantillonnage raisonné de $50 \%$ de maraîchers a été retenu sur la base des critères ci-après : i) disposer de 0,5 ha au moins quelle que soit la source d'eau (puits ou forage) utilisée ; ii) être exploitant d'une parcelle ayant au moins une année de production maraîchère sous irrigation; iii) être en activité sur le site au moment de l'enquête ; iv) se faire recenser une seule fois. Ceci concerne les maraîchers qui disposent de plus d'une exploitation dans la zone d'étude.

\section{Méthode de collecte des données}

Le questionnaire a été l'outil principal utilisé au cours des enquêtes basées sur les entretiens individuels et structurés avec des foci groupes. Ces entretiens ont permis entre autres d'aborder les préoccupations suivantes: fertilisation des sols, traitement phytosanitaire, fréquences d'arrosage, superficie emblavée par saison et annuellement, superficie disponible par exploitation, production, rendement, perception des maraîchers sur la qualité des eaux d'arrosage, règles d'hygiènes observées par la population, etc.

Les eaux d'arrosage ont été prélevées à partir d'un forage ou d'un puits traditionnel dans des flacons stériles dans les sites retenus au niveau des six villages d'étude et dans le village témoin. Avant toute opération de prélèvement, l'eau du forage est pompée pendant au moins une minute afin de s'assurer de ne prélever que l'eau de la nappe après vidange de la colonne, qui peut être susceptible de modification sur le plan physicochimique par rapport à celle de la nappe. Quant à l'eau du puits, elle est directement collectée avec un seau. Il faut signaler que, le seau communément utilisé par les populations est en plastique ou en caoutchouc. Les flacons utilisés sont rincés plusieurs fois avec ladite eau avant leur remplissage total, tout en évitant d'emprisonner les bulles d'air. Sur chaque flacon de prélèvement sont marquées les informations suivantes: Identité du préleveur, date et heure de prélèvement, localité de prélèvement, numéro de l'échantillon et les coordonnées géographiques du lieu de prélèvement. Au total, trois prélèvements ont été faits par site.

\section{Méthodes d'analyse des échantillons}

Toutes les analyses sont faites par le laboratoire de la Direction Générale de l'Eau (DG Eau) à Cotonou. Deux (2) types d'analyse sont réalisés sur les échantillons d'eau à savoir: la détermination de la concentration en nitrates par la méthode au salicylate de sodium et celle des paramètres bactériologiques ci-après : Coliformes totaux et coliformes fécaux conformément aux indications des normes NFT 90-413, NF V 08 - 015, NF V 08 -017; germes totaux selon les prescriptions des normes NFT 
90401 ; Streptocoques fécaux sur le milieu SLANETZ .

\section{Traitement des données}

Pour les besoins de l'étude, les données collectées sur la fertilisation des sols et les règles d'hygiènes ont été traitées à l'aide du programme statistique SAS (Statistic Analysis System) tandis que l'analyse de la variance à 2 ou 3 critères de classification $\left(\mathrm{ANOVA}_{2}\right)$ a été utilisée pour les résultats des analyses d'eau. La comparaison des moyennes a été faite avec le Test Student, Newman-Keuls au seuil de 5\%.

\section{RESULTATS}

Evolution des teneurs en nitrates dans les eaux de la commune de Grand-Popo

Les résultats des analyses effectuées (Figure 3) montrent que les eaux d'arrosage des six villages d'études ont des concentrations dépassant celle de $45 \mathrm{mg} / \mathrm{l}$ prévue par les Directives de Qualité de l'OMS pour l'eau de boisson. Dans le village témoin, la concentration en nitrates est de 4,4 $\mathrm{mg} / \mathrm{l}$ soit plus de dix fois moins que celle préconisée par l'OMS. L'analyse statistique n'a révélé aucune différence significative au seuil de 5\% entre les deux Arrondissements (Figure 4) pour les eaux d'arrosage des villages sous irrigation. Il en résulte que suite au phénomène de lessivage enregistré sur les périmètres irrigués, les eaux souterraines de la Commune de Grand-Popo sont fortement polluées par les nitrates dans les localités où se pratique le maraîchage sous irrigation. Ainsi, la plus petite concentration de nitrates $(107,8 \mathrm{mg} / \mathrm{l})$ est obtenue à Agoué 1 et la plus élevée $(246,4 \mathrm{mg} / \mathrm{l})$ à Yodo-Condji.

Les résultats consignés dans le Tableau 1, illustrent la forte présence des germes totaux, coliformes totaux, coliformes fécaux et streptocoques fécaux dans tous les échantillons d'eau analysés dans les périmètres maraîchers des Arrondissements de Grand-Popo et Agoué. Il est remarqué que les différents germes sont relativement beaucoup plus nombreux dans les échantillons d'Agoué que dans ceux de Grand-Popo. Dans tous les échantillons d'eau analysés, les paramètres microbiologiques recherchés ont présenté des teneurs très supérieures aux normes de l'OMS qui sont celles en vigueur au Bénin. L'analyse statistique montre que c'est seulement pour les coliformes totaux, qu'il existe une différence significative au seuil de $5 \%$ entre les deux Arrondissements.

\section{Evolution des concentrations en bactéries fécales dans les eaux d'irrigation}

Le Tableau 1 présente les concentrations en germes totaux, coliformes totaux, coliformes fécaux et streptocoques fécaux dans la Commune de Grand-Popo.

Tableau 1 : Teneur en germes microbiologiques des eaux d'irrigation de la Commune de Grand-Popo.

\begin{tabular}{|c|c|c|c|c|c|}
\hline $\begin{array}{c}\text { Arrondi } \\
\text { ssements }\end{array}$ & $\begin{array}{c}\text { Villages des } \\
\text { prélèvements }\end{array}$ & $\begin{array}{c}\text { Germes totaux } \\
/ \mathrm{ml}\end{array}$ & $\begin{array}{c}\text { Coliformes } \\
\text { totaux / } 100 \mathrm{ml}\end{array}$ & $\begin{array}{c}\text { Coliformes } \\
\text { fécaux / } 100 \mathrm{ml}\end{array}$ & $\begin{array}{l}\text { Streptocoques } \\
\text { fécaux } / 100 \mathrm{ml}\end{array}$ \\
\hline \multirow{4}{*}{$\begin{array}{l}\text { GRAND- } \\
\text { POPO }\end{array}$} & Ewé-Condji & 90 & 165 & 75 & 200 \\
\hline & Yodo-Condji & Incomptable & 105 & 15 & 55 \\
\hline & Nikoué-Condji & 88 & 50 & 26 & 38 \\
\hline & Hounsoukouè & 98 & 350 & 195 & 200 \\
\hline \multirow{3}{*}{ AGOUE } & Zogbédji & Incomptable & Incomptable & 1320 & 840 \\
\hline & Ayi-Guinnou & 201 & 405 & 250 & 22 \\
\hline & Agoué 1 & 102 & 299 & 205 & 160 \\
\hline \multirow[t]{2}{*}{ NORMES } & OMS & $\begin{array}{l}\text { Absence dans } \\
\text { l'eau }\end{array}$ & $\begin{array}{l}\text { Absence dans } \\
\text { l'eau }\end{array}$ & $\begin{array}{l}\text { Absence dans } \\
\text { l'eau }\end{array}$ & $\begin{array}{l}\text { Absence dans } 50 \\
\mathrm{ml}\end{array}$ \\
\hline & BENIN & $\begin{array}{l}\text { Absence dans } \\
\text { l'eau }\end{array}$ & $\begin{array}{l}\text { Absence dans } \\
\text { l'eau }\end{array}$ & $\begin{array}{l}\text { Absence dans } \\
\text { l'eau }\end{array}$ & $\begin{array}{l}\text { Absence dans } \\
\text { l'eau }\end{array}$ \\
\hline
\end{tabular}

Source: Laboratoire de la Direction Générale de l'Eau, 2008 ; OMS = Organisation Mondiale de la Santé. 


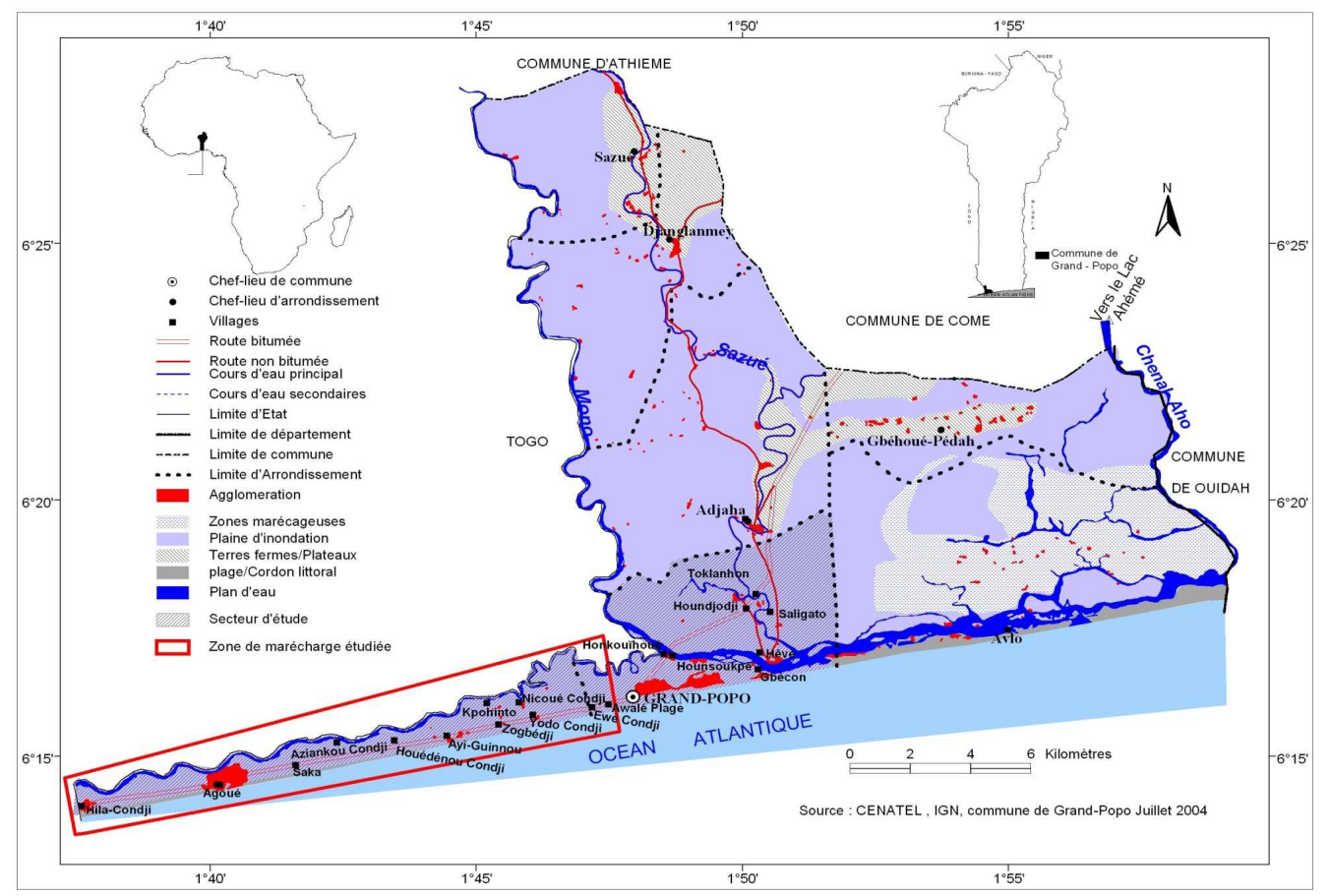

Figure 1: Situation géographique du secteur d'étude.

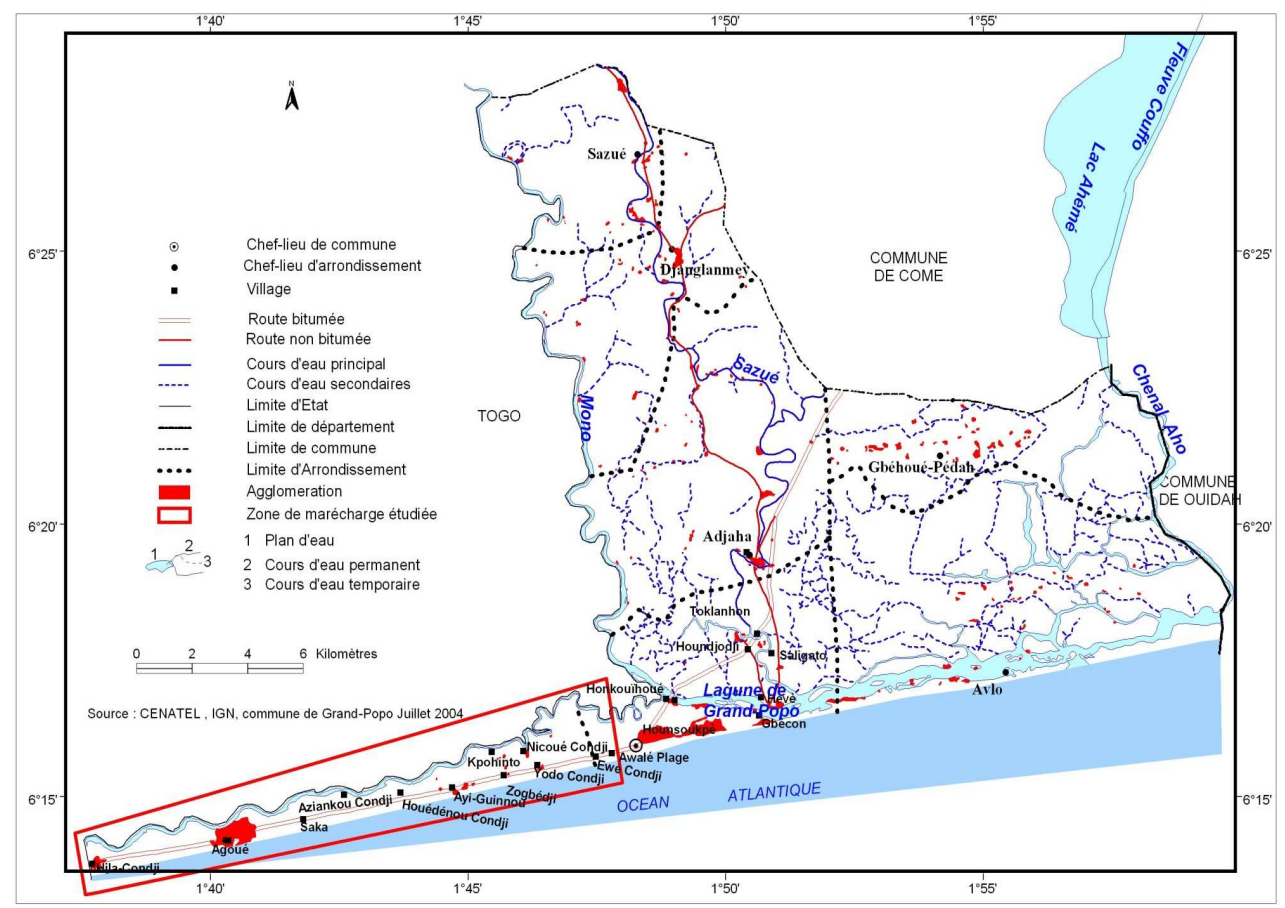

Figure 2: Complexe hydrographique de la Commune de Grand-Popo. 


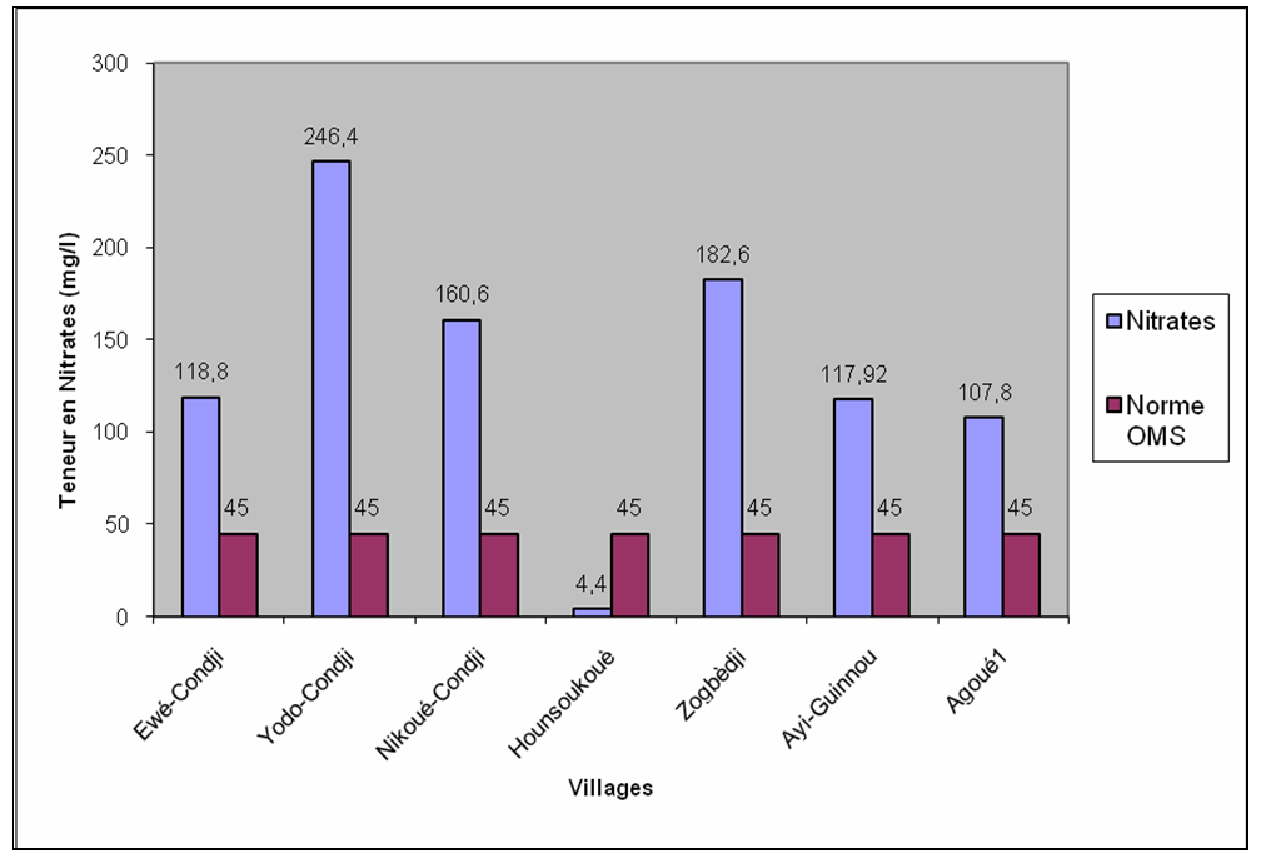

Figure 3 : Teneur des nitrates (mg/l) dans les eaux d'irrigation dans la Commune de Grand-Popo (Source : Laboratoire de la Direction Générale de l'Eau, 2008). OMS = Organisation Mondiale de la Santé.

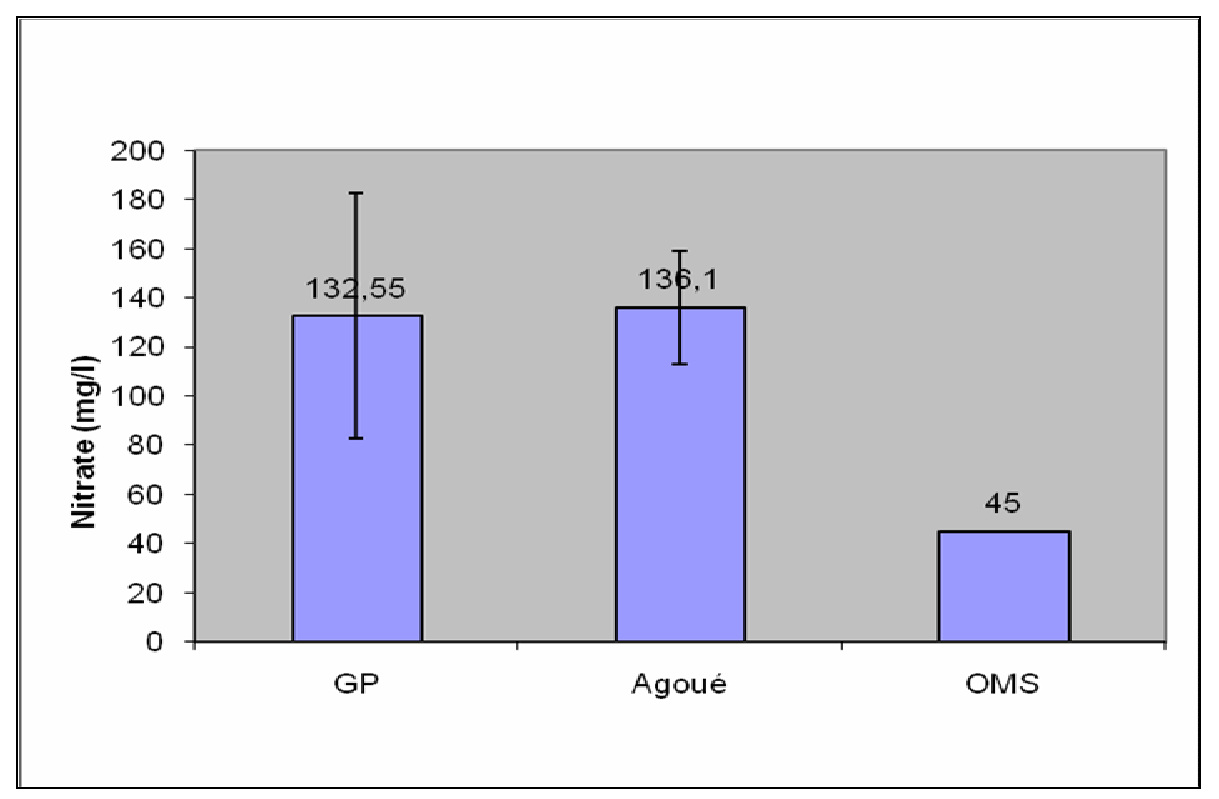

Figure 4 : Teneur des nitrates $(\mathrm{mg} / \mathrm{l})$ dans les eaux des arrondissements d'Agoué et de Grand-popo. GP = Grand - Popo, OMS = Organisation Mondiale de la Santé. 


\section{DISCUSSION}

\section{Des résultats troublants}

D'une manière générale, au niveau des deux Arrondissements et plus spécifiquement dans les zones de maraîchage sous irrigation, la teneur en nitrates a atteint $246,4 \mathrm{mg} / \mathrm{l}$ à Yodo-Condji, soit plus de 5 fois le seuil de 45 $\mathrm{mg} / \mathrm{l}$ préconisé par l'OMS tandis qu'il n'est que de 4,4 mg/l à Hounsoukouè, village où le maraîchage se fait sans irrigation. Ce dernier résultat a été confirmé par de récentes analyses d'eaux de puits et de forages, réalisées en 2005 par le laboratoire de la Direction Générale de l'Eau, dans les arrondissements de Djanglanmey, Sazué et Adjaha, Commune de Grand-Popo, où se pratique le maraîchage sans irrigation et où les taux de nitrates obtenus sont dans l'ordre de 14 à $32 \mathrm{mg} / \mathrm{l}$. Il en est de même de celles réalisées de 2004 à 2008 par la même structure, dans les Communes d'Athiémé et de Comè où l'on a obtenu des taux de nitrates variant entre 0 et $6 \mathrm{mg} / \mathrm{l}$. Par conséquent, il se dégage de nos résultats que les eaux d'irrigation des périmètres maraîchers des Arrondissements de Grand-popo et d'Agoué sont polluées par les nitrates et que le niveau de pollution est plus élevé à Grand-Popo qu'à Agoué. Ces résultats se rapprochent de ceux de Makoutodé et al. (1999) à Grand-Popo où $50 \%$ des eaux de puits analysés présentaient déjà des teneurs dépassant le seuil de 45 mg/l préconisé par l'OMS.

L'essentiel de cette pollution est dû à la différence entre les apports en nitrates sous forme d'engrais et ce qui est réellement consommé par les plantes (CNRS, 2005).

Quant aux paramètres bactériologiques, les cas de Yodo-Condji à Grand-Popo et de Zogbédji à Agoué suscitent des inquiétudes au regard des forts taux (nombre incomptable) enregistrés pour l'ensemble. Toutefois, les chiffres obtenus sont dans la même fourchette que ceux obtenus au niveau des eaux des périmètres maraîchers de Cotonou où les teneurs d'après Agbossou et Sanny (2005) indiquent une pollution bactériologique. C'est le même constat qui a été fait par Makoutodé et al. (1999) à Grand-Popo où les taux de coliformes dépassent des milliers de colonies par $100 \mathrm{ml}$. Les travaux de Azonhè (2009) dans la dépression des Tchi au Sud du Bénin ont montré au niveau des eaux de puits et de rivière des résultats similaires.

Obuobie et al. (2006) dans une étude réalisée au Ghana en 2006 sur la qualité des eaux d'arrosage utilisées sur les périmètres maraîchers urbains et péri urbains est parvenu aux résultats du même ordre, aussi bien du point de vue des teneurs en nitrates (45 à 60 $\mathrm{mg} / \mathrm{l})$ que des paramètres microbiologiques dont les teneurs dépassent des dizaines de milliers de colonies. D'après Cissé et al. (2002) dans une étude réalisée à Ouagadougou, le constat est le même pour les niveaux de pollution des eaux d'arrosage dans les sites maraîchers autour des eaux de barrage.

Nos résultats sont conformes à ceux rapportés par les différents travaux cités cidessus. Face au constat de la pollution des eaux dans la zone d'étude, il s'avère indispensable d'en rechercher les causes afin de limiter les déconvenues.

\section{Origines anthropiques des pollutions}

La forte teneur obtenue en nitrates et en paramètres microbiologiques (germes totaux, coliformes totaux, de coliformes fécaux et de streptocoques fécaux) dans les villages d'études provient de la contamination des eaux d'irrigation par diverses activités humaines connexes ou non à l'irrigation des cultures maraîchères dans la Commune de Grand-Popo. En effet, les forts taux obtenus pour les nitrates et les bactéries s'expliquent non seulement par leur présence naturelle dans les eaux souterraines, au regard de la richesse en minéraux et microorganismes des couches sablonneuses traversées par l'eau au cours de son infiltration, mais surtout par les 
actions de l'homme et des animaux sur ces sols. Il s'agit entre autres de :

- la fertilisation des sols par les engrais chimiques et organiques ;

- la défécation sur les sites par les populations et les troupeaux en divagation;

- le déversement des déchets divers dans le milieu.

\section{Fertilisation des sols par les engrais chimiques et organiques}

Compte tenu de l'état de pauvreté des sols (sols sableux) de la zone d'étude confirmé par Lawani et al. (2000), les cultures maraîchères ne peuvent y être pratiquées sans apports d'intrants. A Grand-Popo, ce sont les engrais minéraux (urée et NPK) et organiques (bouse de vache et fientes de volaille) qui sont intensément utilisés par les producteurs parce que préoccupés par une bonne productivité et des rendements élevés (Dablaka, 2004). Cette pratique qui consiste à épandre, des doses massives d'engrais azotés (1000 - $3000 \mathrm{~kg} / \mathrm{ha}$ de NPK et 2500 - $5000 \mathrm{~kg} / \mathrm{ha}$ d'urée au lieu de $400 \mathrm{~kg}$ et $200 \mathrm{~kg} / \mathrm{ha}$ pour respectivement chacun d'eux) contraires à ce qui est prescrit par les Fiches Techniques de l'Institut National de la Recherche Agricole (I.N.R.A.B), a pour conséquence la pollution des sols et voire celle des eaux et des plantes. En effet, conformément aux résultats des analyses, les eaux du village témoin (Hounsoukouè) où le maraîchage se fait sans irrigation et sans apport de fumure ne sont pas exposées aux nuisances des engrais apportés aux sols des sites maraîchers. En effet, le taux de $4,4 \mathrm{mg} / \mathrm{l}$ enregistré est largement en dessous du seuil de $45 \mathrm{mg} / \mathrm{l}$ exigé par l'OMS.

Normalement, en l'absence de contamination anthropique, la teneur des eaux souterraines est de l'ordre de 0,1 à $1 \mathrm{mg} / \mathrm{l}$ (C.N.R.S, 2005). Mais aujourd'hui, avec le développement de l'agriculture urbaine et péri urbaine, cette fourchette est souvent dépassée dans certaines localités au regard du type de fumure utilisé. En effet, les teneurs en nitrates obtenues à l'issue des travaux similaires réalisés par Agbossou et Sanny (2005) au niveau des eaux d'irrigation des périmètres maraîchers de Cotonou (Houéyiho et Kouhounou) sont de 0,00 à $3,9 \mathrm{mg} / \mathrm{l}$. Le compost est plus utilisé sur ces sites que les engrais minéraux.

Les nitrates sont des composés chimiques faits d'azote et d'oxygène qui se trouvent à l'état naturel dans certains légumes, dans les viandes et dans le sol. Il y en a aussi dans les engrais commerciaux, les déjections animales et les excrétas humains, (M.A.F., 2001). Dans le sol, ils proviennent d'après Leclerc (2008), de la décomposition de la matière organique animale ou végétale par les microorganismes. Ils sont ensuite utilisés par les plantes pour leur croissance et transformés en protéines végétales indispensables à la vie humaine et animale. Mais lorsque cette capacité d'absorption est dépassée, que le cycle biologique naturel est ainsi rompu, les nitrates en excès rejoignent les ressources en eau superficielles (rivières, fleuves, lacs...) par ruissellement ou s'infiltrent dans le sol et menacent les ressources souterraines. Les eaux sont alors polluées par cet excédent de nitrates, qui constituent l'une des causes majeures de la dégradation des eaux naturelles à long terme. En outre, d'après le même auteur, la quantité de nitrates disponibles dans le sol est le principal facteur limitant la croissance des plantes. Ce besoin en nitrates et autres ions majeurs est comblé par amendement des sols avec les engrais chimiques et organiques disponibles sur les marchés Béninois et Togolais. Selon les résultats d'enquête, plus de $60 \%$ des maraîchers appliquent la fumure au moins quatre fois au cours du cycle végétatif des cultures avec des doses (1000-3000 kg/ha de NPK et 2500-5000 kg/ha d'urée) supérieures à celles recommandées par les services techniques.

Quant aux engrais organiques (bouse de vache et fiente de volaille), ils sont 
répandus sur les sols et mélangés à eux pendant la confection des planches. De par leur origine métabolique (déjections animales), ces matières organiques en dehors des germes bactériologiques, donnent assez de nitrates lors de la minéralisation (M.A.F., 2001).

\section{Défécation sur les sites et déversement des déchets dans le milieu}

Du fait de l'insuffisance de latrines dans la zone d'étude, plus de $70 \%$ de la population défèquent dans la brousse, à l'air libre sur les sites d'étude ou à proximité et le long des cours d'eau et de la plage. Il en est de même des troupeaux de bœuf ou de petits ruminants en quête de fourrage sur les sites en jachère ou en attente d'être mis en valeur. Ces mauvaises habitudes des populations de Grand-Popo, dues à une insuffisance d'assainissement du milieu d'étude, sont également à la base des pollutions des eaux. Les résultats de l'étude menée par Atinkpahoun (2004) dans les zones lacustres confirment que les mauvaises habitudes des populations et le manque d'assainissement du milieu sont responsables des pollutions mentionnées. Abondant dans le même sens, Makoutodé et al. (1999) ont justifié la pollution des puits de Grand-Popo par les nitrates et germes bactériologiques par le faible niveau d'assainissement et de l'hygiène publique et les mauvais comportements de la communauté comme la défécation à l'air libre. D'autres mauvaises habitudes entretenues par les populations des villages d'étude sont le déversement direct dans le milieu des déchets solides et liquides. A ce titre, on peut citer les matières plastiques, les boîtes vides de conserves, les débris végétaux, les cadavres d'animaux et toutes sortes de déchets ménagers. Les tas d'immondices sont perceptibles et nombreux dans les villages d'Ayi-Guinnou et Zogbédji en dehors des déchets solides, liquides et ménagers qui sont déversés çà et là dans la Commune. La pluie et les eaux de ruissellement sont responsables de la dissémination des polluants dans l'environnement (C.N.R.S., 2005).

Maire et al. (1994) affirment: «il a rarement été prouvé que les aquifères africains eux-mêmes soient contaminés. La majorité des sources convenablement captées donnent des eaux qui ne contiennent pratiquement pas de coliformes fécaux. Une telle contamination n'est cependant pas exclue dans les régions très riches en alluvions grossières et surtout en milieu karstique ». Il en résulte donc que l'utilisation de la nature comme site d'évacuation des excrétas et le rejet des ordures dans les végétations bordières des villages participent à la contamination des eaux de consommation par ruissellement/ infiltration et même dans certains cas par contamination passive avec les animaux domestiques en divagation. C'est ce qui explique d'ailleurs les fortes teneurs enregistrées dans ces localités pour les germes microbiologiques provenant des excrétas d'origines humaine et animale, outre les concentrations non négligeables obtenues pour les nitrates.

\section{Conclusion}

A Grand-Popo, le maraîchage sous irrigation et certaines mauvaises habitudes de la population contribuent énormément aux pollutions chimique et microbiologique des eaux souterraines. Cette situation est plus dramatique que les résultats auxquels a abouti une étude similaire menée à Cotonou (Agbossou et Sanny, 2005) pour ce qui est des nitrates, et presque identique à ceux obtenus pour les germes microbiologiques. Les concentrations excessives des nitrates tournent autour d'une moyenne de 132,6 mg/l à GrandPopo tandis qu'elle est de 136,1 mg/l à Agoué. Quand aux bactéries d'origine fécale, les teneurs obtenues dépassent exagérément les normes admises dans les deux arrondissements. Dans la zone d'étude, la pollution chimique (par les nitrates) provient 
de l'utilisation excessive d'engrais minéraux (NPK et urée) et fientes de volaille en fonction de leur degré de minéralisation tandis que la pollution microbiologique est engendrée par les actions conjuguées des excrétas humains et animaux dont notamment la volaille, les bœufs et les porcs.

La mer et plus particulièrement le littoral, apparaissent comme le réceptacle de tous les polluants d'origine tellurique, fluviale ou marine. La qualité des eaux du littoral est localement préoccupante et seule une politique globale peut remédier à la dégradation progressive du milieu marin. Un effort est fait à l'échelon européen qui se manifeste par des recommandations, puis des directives contraignantes qui visent à limiter les sources de pollution: limitation de la pollution agricole, traitement plus poussé des eaux usées urbaines, des effluents industriels, des émissions aériennes polluantes, des émissions des moteurs à combustion interne (imbrûlés des diesels, super sans plomb). En Afrique en général et plus particulièrement au Bénin, beaucoup restent à faire notamment, la construction des infrastructures d'assainissement et la sensibilisation des populations sur les dangers que revêt la pollution des eaux, des sols et des légumes à partir des activités agricoles ou de certaines pratiques malséantes et les dispositions à prendre par elles pour les surmonter.

\section{REMERCIEMENTS}

La présente étude n'aurait pas pu être réalisée sans l'assistance financière du Laboratoire d'Hydraulique et de Maîtrise de l'Eau de la Faculté des Sciences Agronomiques (FSA) de l'Université d'Abomey-Calavi (UAC), à travers le Projet NPT 145.

\section{REFERENCES BIBLIOGRAPHIQUES}

Agbossou E, Sanny S. 2005. Etude des Biocontaminants et Migration des Agents Toxiques dans les Cultures Maraîchères au Bénin. Agence Béninoise pour l'Environnement : Cotonou, Bénin ; 85.

Atidégla S. 2005. Atouts et contraintes des modes d'irrigation dans les exploitations urbaines et péri urbaines de la Commune de Grand-Popo. Mémoire de DEA, EDP/FLASH, Université d'AbomeyCalavi (UAC), p.140.

Atinkpahoun C. 2004. L'évaluation de la pollution inorganique azotée et phosphorée dans les écosystèmes du lac Nokoué et du Chenal de Cotonou et l'identification des différentes sources ». Bulletin d'informations de l'ABPEE, $\mathrm{N}^{\circ}$ 004, Cotonou, Bénin, p.3.

Azonhè T. 2009. Analyse systémique des déterminants environnementaux de la morbidité paludique et diarrhéique chez les populations du secteur agricole dans la dépression des Tchi au sud du Bénin. Thèse de Doctorat, EDP/FLASH, Université d'Abomey-Calavi (UAC), p. 196.

Cissé G, Kientga M, Ouédraogo B, Tanner M. 2002. Impact sanitaire et nutritionnel des hydro-aménagements en Afrique. Développement du maraîchage autour des eaux de barrage à Ouagadougou : quels sont les risques sanitaires à prendre en compte ? Base Documentaire de la Santé Publique, 11(1): 31-38.

C.N.R.S. (Centre National de Recherche Scientifique) 2005. L'irrigation et les dégradations. Document scientifique sur l'eau ; 7-9.

Dablaka, 2003. Rapport de stage sur l'Etude de la rentabilité de l'irrigation dans le département du Mono au Bénin : Village d'Ayi Guinnou, IUT Amiens, p. 30.

Dossa SJ, Mensah GA, Laleye AP. 2007. Evaluation de l'impact des activités humaines sur la survie des tortues marines sur la côte de Grand-Popo: Problématique et proposition d'aménagement de la zone. Bulletin 
d'information de l'ABPEE, N ${ }^{\circ}$, Juin 2007, Cotonou, p. 7.

I.N.S.A.E. (Institut National de la Statistique et de l'Analyse Economique), 2004. Cahier des villages et quartiers de ville, Département du Mono, MPPD, Cotonou, p. 25 .

Lawani M, Lawani L, Akakpo A, Déguénon S. 2005. Rapport sur la reconnaissance pédologique sur le Littoral à Grand-Popo. Centre National d'Agro-Pédologie, Abomey-Calavi, Bénin, p. 26.

Leclerc H. 2008. Nitrates de l'eau d'alimentation et risques pour la santé ». Académie de l'Agriculture de France, 14 ; 1-3. Site web, www.inst.elevage.asso.fr

M.A.E.P. (Ministère de l'Agriculture, de l'Elevage et de la Pêche) 2005. Annuaire statistique, Production végétale, Campagne 2003-2004, Cotonou, DPP., p.426.
M.A.F. (Manitoba Agriculture and Food) 2001. Les nitrates dans le sol et dans l'eau ; 2-6. Site web, www.gov.mb.ca

Maire R, Pomel S, Salomon JN. 1994. Enregistreurs et Indicateurs de l'Environnement en Zone Tropicale. Presses Universitaires de Bordeaux ; 492.

Makoutodé M, Assani AK, Ouendo E-M, Agueh VD, Diallo P. 1999. Qualité et mode de gestion de l'eau de puits en milieu rural au Bénin: Cas de la sous préfecture de Grand-Popo. Médecine d'Afrique Noire, 46: 4.

Obuobie E, Keraita B, Danso G, Amoa P, Cofie OO, Raschid-Sally L, Pay D. 2006. Irrigated Urban Vegetable Production in Ghana: Characteristics, Benefits and Risks. IWMI-RUAF-CPWF: Accra, Ghana; 150. 